\title{
Vezatin regulates seizures by controlling AMPAR-mediated synaptic activity
}

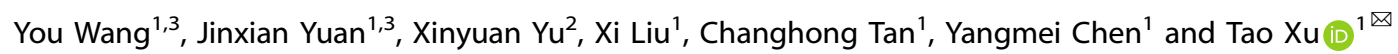 \\ (c) The Author(s) 2021
}

\begin{abstract}
Although many studies have explored the mechanism of epilepsy, it remains unclear and deserves further investigation. Vezatin has been reported to be a synaptic regulatory protein involved in regulating neuronal synaptic transmission (NST). However, the role of vezatin in epilepsy remains unknown. Therefore, the aims of this study are to investigate the underlying roles of vezatin in epilepsy. In this study, vezatin expression was increased in hippocampal tissues from pilocarpine (PILO)-induced epileptic mice and a $\mathrm{Mg}^{2+}$ free medium-induced in vitro seizure-like model. Vezatin knockdown suppressed seizure activity in PILO-induced epileptic mice. Mechanistically, vezatin knockdown suppressed AMPAR-mediated synaptic events in epileptic mice and downregulated the surface expression of the AMPAR GluA1 subunit (GluA1). Interestingly, vezatin knockdown decreased the phosphorylation of GluA1 at serine 845 and reduced protein kinase A (PKA) phosphorylation; when PKA phosphorylation was suppressed by $\mathrm{H}-89$ (a selective inhibitor of PKA phosphorylation) in vitro, the effects of vezatin knockdown on reducing the phosphorylation of GluA1 at serine 845 and the surface expression of GluA1 were blocked. Finally, we investigated the pattern of vezatin in brain tissues from patients with temporal lobe epilepsy (TLE), and we found that vezatin expression was also increased in patients with TLE. In summary, the vezatin expression pattern is abnormal in individuals with epilepsy, and vezatin regulates seizure activity by affecting AMPAR-mediated NST and the surface expression of GluA1, which is involved in PKA-mediated phosphorylation of GluA1 at serine 845 , indicating that vezatin-mediated regulation of epileptic seizures represents a novel target for epilepsy.
\end{abstract}

Cell Death and Disease (2021)12:936; https://doi.org/10.1038/s41419-021-04233-2

\section{INTRODUCTION}

Epilepsy is a heterogeneous disease with a complicated etiology and mechanism [1, 2]. Although many studies have explored the underlying mechanism of epilepsy, the etiology and mechanism of epilepsy remain unclear and require further investigation to potentially aid in the development of new disease-modifying therapies to suppress seizures in patients with epilepsy.

Vezatin, a transmembrane protein expressed in the mammalian brain, was found to be enriched in hippocampal neurons in mice and is associated with anxiety-related behaviors in mice $[3,4]$. Previous studies have indicated that vezatin is essential for the formation of cell junctions, which may influence intercellular signal transmission $[5,6]$. Vezatin was found to be expressed at high levels in neuronal dendritic spines and regulates synaptic plasticity in vivo and in vitro [3, 4]. Furthermore, altered expression of vezatin affects glutamate receptor-mediated neuronal synaptic transmission (NST) [3]. NST dysfunction plays a vital role in the pathophysiological mechanism of epilepsy $[2,7]$. Abnormal NST contributes to an imbalance between neuronal excitation and inhibition, further promoting seizure generation and propagation and even epileptogenesis [2, 7]. Therefore, based on the abovementioned functions of vezatin, we hypothesized that vezatin may play a specific role in epilepsy by influencing NST.
Hence, the aim of this study was to investigate the role of vezatin in regulating seizure activity in a mouse model of pilocarpine (PILO)-induced epilepsy and further explore the molecular mechanism by which vezatin regulates seizure activity.

\section{RESULTS \\ Expression pattern of vezatin in epileptic models in vivo and in vitro}

We investigate the expression pattern of vezatin in a mouse model of PILO-induced epilepsy. Hippocampal local-field potentials (LFPs) (Fig. 1a, b) were recorded to confirm the induction of PILO-induced epilepsy in mice. Immunofluorescence staining showed that vezatin (green) expressed in the hippocampal CA1 region in mice colocalized with the neuronal marker microtubuleassociated protein 2 (MAP2) (red) (Fig. 1c); the mean fluorescence intensity (MFI) of vezatin was higher in the epilepsy group than in the control group (especially in the stratum radiatum), indicating increased expression of vezatin in the epilepsy model (Fig. 1d). The further western blotting analysis confirmed that the expression of vezatin was increased in the epilepsy group (Fig. 1e, f).

In cultured hippocampal neurons, immunofluorescence staining showed that vezatin (green) was expressed in neurons positive for the neuronal marker beta-Tubulin III (Tubb3) (red) and colocalized

\footnotetext{
${ }^{1}$ Department of Neurology, the Second Affiliated Hospital of Chongqing Medical University, 400010 Chongqing, China. ${ }^{2}$ Department of Neurology, Chongqing Hospital of Traditional Chinese Medicine, 400021 Chongqing, China. ${ }^{3}$ These authors contributed equally: You Wang, Jinxian Yuan. Edited by Joern Steinert ${ }^{\bowtie}$ email: xutao@hospital.cqmu.edu.cn Edited by Joern Steinert
}

Received: 30 May 2021 Revised: 20 September 2021 Accepted: 28 September 2021

Published online: 12 October 2021 
PILO-induced epileptiform discharge

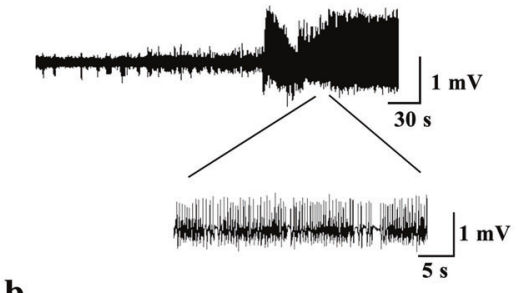

b

Spontaneous epileptiform discharge

baseline

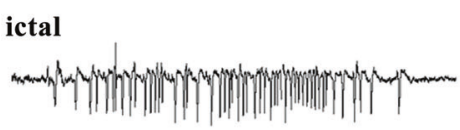

interictal
C $\mathbf{C t}_{\mathbf{r}}$
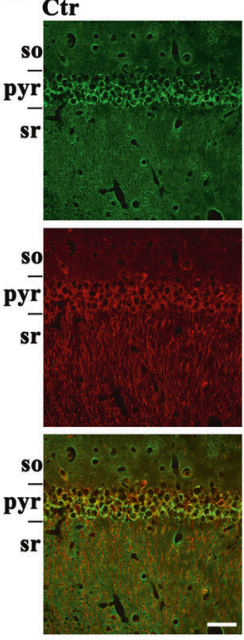

$\mathbf{e}$
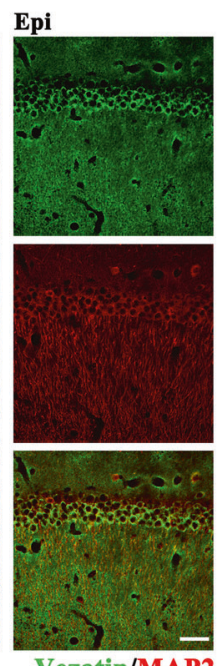

Vezatin/MAP2

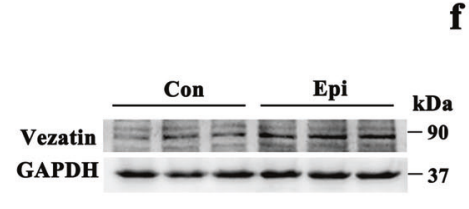

d
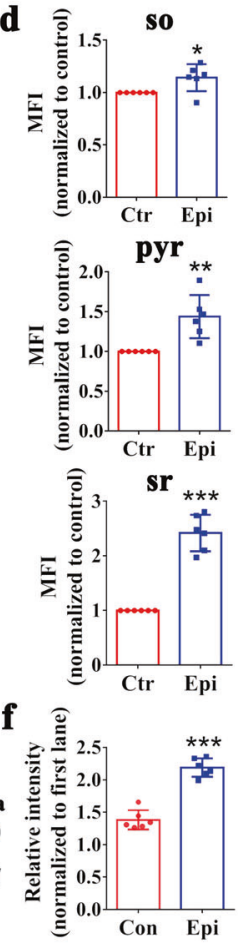

Fig. 1 The pattern of vezatin expression in the PILO-induced epilepsy model. a Representative image of PILO-induced epileptiform discharges in the acute stage and (b) representative image of spontaneous epileptiform discharges in the chronic stage. $\mathbf{c}$ Representative images of immunofluorescence staining demonstrating that in both the control group and epilepsy group, vezatin (green) was expressed in the hippocampal CA1 region, which was divided into three layers: the stratum oriens (so), pyramidal cell layer (pyr), and stratum radiatum (sr). Vezatin (green) was colocalized with the neuronal and dendritic marker MAP2 (red). d Statistical analysis of the MFI of vezatin in the three layers of the CA1 region ( $n=6$ per group; so, $P=0.043$; pyr, $P=0.003$; sr, $P<0.001$ ). e Representative images of western blots showing hippocampal vezatin expression in the control (Con) group and epilepsy (Epi) group and (f) the corresponding statistical analysis $(n=6$ per group, $P<0.001)$. Student's $t$ test; ${ }^{*} P<0.05$, ${ }^{* *} P<0.01$, and ${ }^{* * *} P<0.001$.

with the excitatory postsynaptic marker postsynaptic density protein 95 (PSD-95) (purple) (Fig. 2a) but not with the presynaptic marker synapsin 1 (purple) (Fig. 2b). Immunofluorescence staining indicated a higher fluorescence intensity of vezatin in the $\mathrm{Mg}^{2+}$ free medium-induced in vitro seizure-like model than in the control group (Supplementary Fig. S1). Furthermore, western blot analysis also indicated increased expression of vezatin in the in vitro seizure-like model group (Supplementary Fig. S1).

Lentivirus-mediated knockdown of vezatin in vivo and in vitro After 2 weeks of lentiviral vectors (LVs) injection into the hippocampal CA1 region of mice, immunofluorescence staining showed the LVs (green) were widely distributed in the hippocampal CA1 region, indicating successful delivery of the LVs to this region (Supplementary Fig. S2). Western blot analysis showed a lower expression level of vezatin in the shRNA group than that in the Scr-seq group at 2 and 4 weeks after LVs injection (Supplementary Fig. S2), indicating that the LVs expressing the shRNA targeting vezatin reduced the expression of vezatin in the mouse hippocampal region.

In primary cultured hippocampal neurons, the LVs successfully infected neurons on day in vitro (DIV) 10 (Supplementary Fig. S3). Western blot analysis revealed a lower expression level of vezatin in the shRNA group than that in the Scr-seq group (Supplementary Fig. S3), indicating that the LVs expressing the shRNA targeting vezatin reduced vezatin expression in cultured neurons.

\section{The role of vezatin in regulating seizure activity}

Vezatin knockdown in the hippocampal CA1 region of a mouse model of PILO-induced epilepsy prolonged the SRS latency and reduced the number of SRSs per week and the proportion of stage
4-5 convulsive SRSs (Fig. 3a-c), indicating that vezatin knockdown suppressed seizure activity in epilepsy model. Moreover, hippocampal LFP recordings showed that the number of seizure-like events (SLEs) per 30 min in the shRNA group was lower than that in the Scr-seq group (Fig. 3d, e), indicating that vezatin knockdown inhibited epileptiform discharges in a mouse model of PILO-induced epilepsy. In the in vitro seizure-like model, the frequency of spontaneous epileptiform discharges (SEDs) was lower in the shRNA group than in the Scr-seq group (Supplementary Fig. S4), indicating that vezatin knockdown reduced epileptiform discharges in an in vitro seizure-like model.

\section{Role of vezatin in the regulation of NST}

Due to the colocalization of vezatin with the excitatory postsynaptic marker PSD-95 and the role of vezatin in regulating seizures, we postulated that vezatin plays a role in regulating NST in epilepsy and investigated the underlying mechanism. After seizures were recorded, mice were sacrificed to obtain whole-cell patch-clamp recordings. First, we measured glutamatergic NST, including a-amino-3-hydroxy-5-methyl-4-isoxazolepropionic acid receptor (AMPAR)-mediated and $\mathrm{N}$-methyl-d-aspartate receptors (NMDAR)-mediated NST, in hippocampal CA1 neurons of epileptic mice. AMPAR-mediated miniature excitatory postsynaptic current (AMPAR-mEPSC) recordings revealed that vezatin knockdown reduced the amplitude of AMPAR-mEPSCs but did not alter the frequency of AMPAR-mEPSCs (Fig. 4a, b). No significant differences in the amplitude and frequency of NMDAR-mediated mEPSCs (NMDAR-mEPSCs) were observed between the Scr-seq group and shRNA group (Fig. 4c, d). Moreover, we obtained miniature inhibitory postsynaptic current (mIPSC) recordings to investigate the role of vezatin in regulating inhibitory synaptic transmission in 

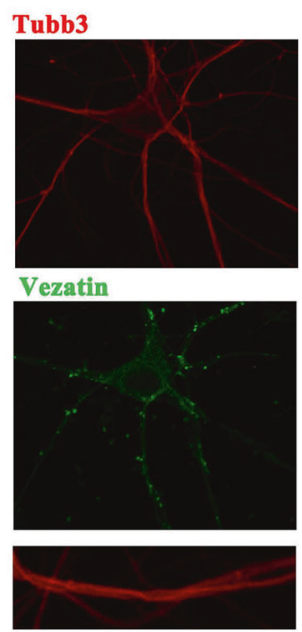

$\mathbf{5}$

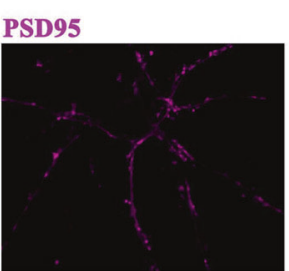

Merge
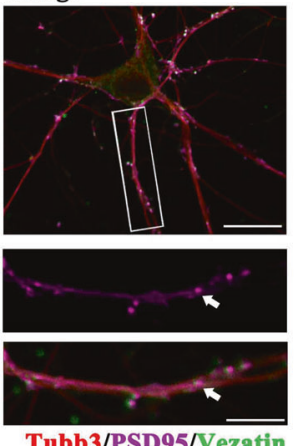

b

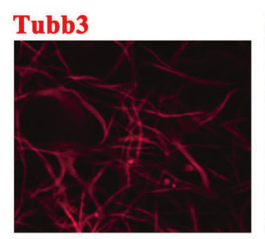

Synapsin 1

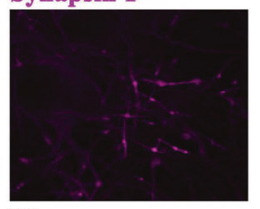

Merge
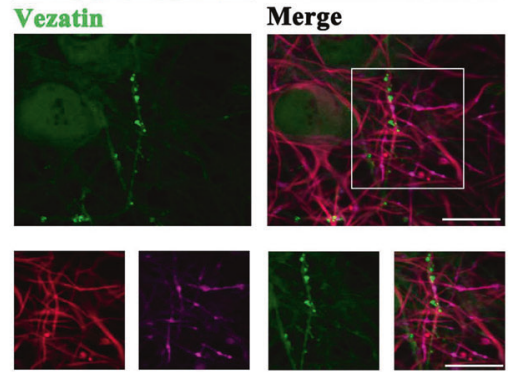

Tubb3/Synapsin 1/Vezatin

\section{Tubb3/PSD95/Vezatin}

Fig. 2 The pattern of vezatin expression in hippocampal neurons in vitro. Representative images of immunofluorescence staining demonstrating that vezatin (green) was localized in neurons positive for the neuronal marker Tubb3 (red) and colocalized with the excitatory postsynaptic marker PSD-95 (purple) (a) but not with the excitatory presynaptic marker synapsin 1 (purple) (b).

$\mathbf{a}$

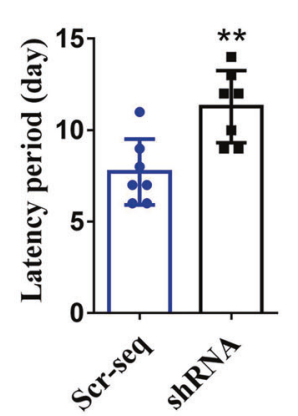

d

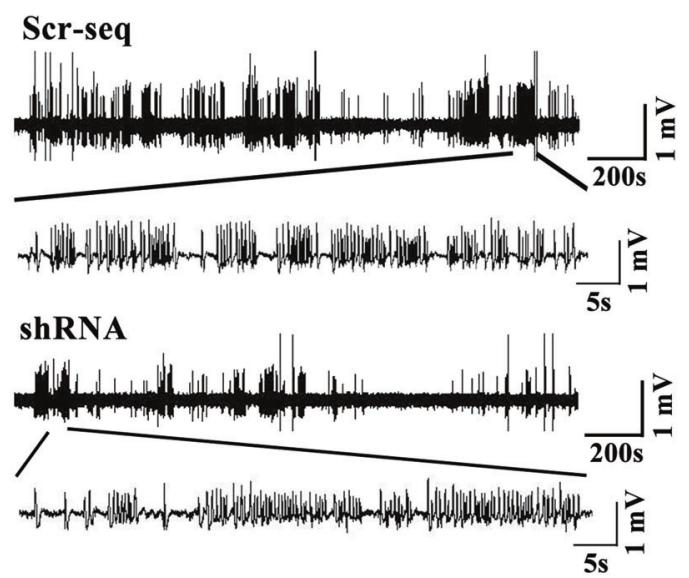

C

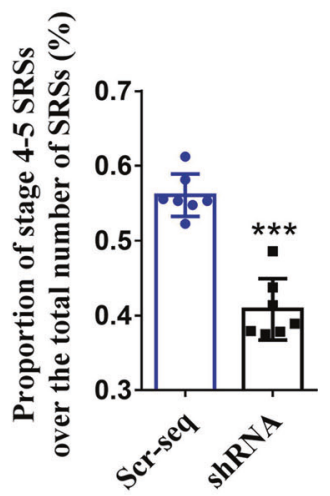

e

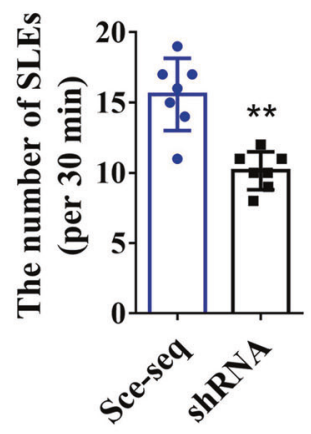

Fig. 3 The effect of vezatin knockdown on seizure activity in a mouse model of PILO-induced epilepsy. a Statistical analysis of the spontaneous recurrent seizures (SRSs) latency $(P=0.004)$, b the number of SRSs (seizures/week) $(P=0.002)$, and $\mathbf{c}$ the proportion of Racine stage 4-5 SRSs relative to the total number of SRSs $(\%)(P<0.001)$. $\mathbf{d}$ Representative images comparing hippocampal LFP recordings between the Scr-seq group and shRNA group and (e) the corresponding statistical analysis of the number of SLEs per 30 min, as identified from hippocampal LFP recordings $\left(n=7\right.$ per group, $P=0.003$ ). Student's $t$ test; ${ }^{*} P<0.05,{ }^{* *} P<0.01$, and ${ }^{* * *} P<0.001$. 
a

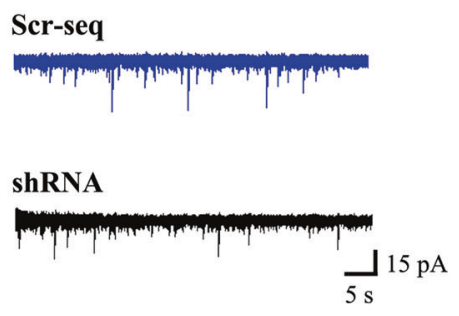

c

Scr-seq

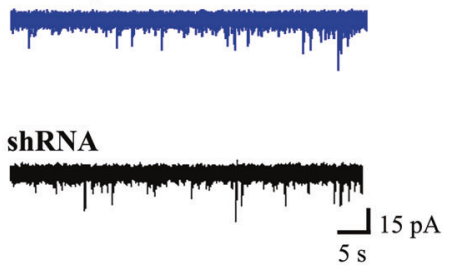

e Scr-seq

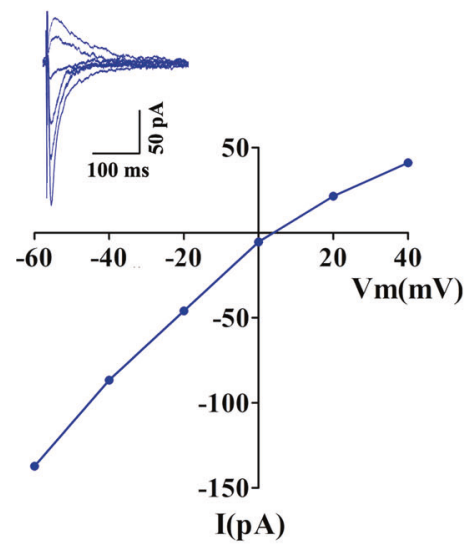

g

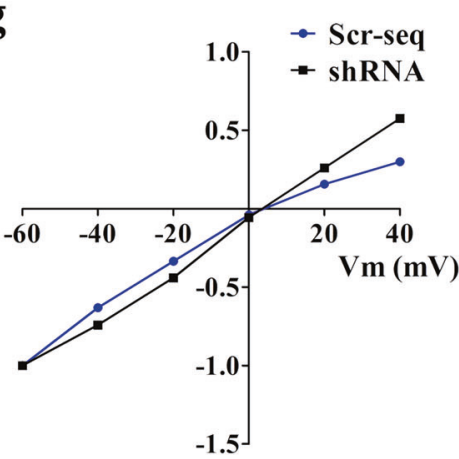

Normalized peak b

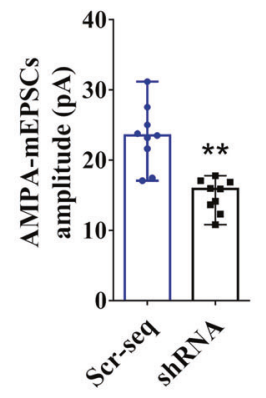

d
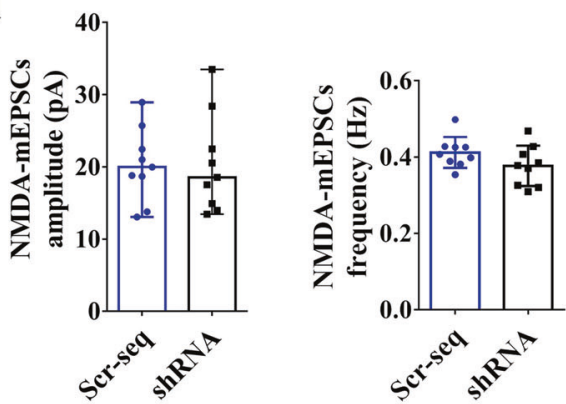

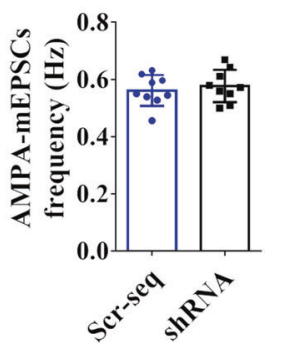

sect 
Fig. 4 The effect of vezatin knockdown on AMPAR- and NMDAR-mediated synaptic currents in the hippocampal CA1 neurons of epileptic mice. a Representative images of AMPAR-mEPSCs in the Scr-seq group and shRNA group and (b) the corresponding statistical analysis of the amplitude (presented as medians and ranges; $P=0.003$; Kruskal-Wallis test; $\left.{ }^{*} P<0.01\right)$ and frequency of AMPAR-mEPSCs $(n=9$ per group). c Representative images of NMDAR-mEPSCs in the Scr-seq group and shRNA group and (d) the corresponding statistical analysis of the amplitude (presented as medians and ranges; Kruskal-Wallis test) and frequency of NMDAR-mEPSCs ( $n=9$ per group). Current-voltage (I-V) relationships obtained by plotting the amplitudes of AMPAR-eEPSCs at holding potentials ranging from $-60 \mathrm{mV}$ to $+40 \mathrm{mV}$ in the Scr-seq group (e) and in the shRNA group (f). The normalized I-V curves of AMPAR-eEPSCs (normalized to the amplitude evoked at -60 mV) (g) and statistical analysis of the Scr-seq group and shRNA group (h). The RI was calculated from the I-V curves as the ratio of the AMPAR-eEPSC amplitude at $-60 \mathrm{mV}$ to the AMPAR-eEPSC amplitude at $+40 \mathrm{mV}\left(n=8\right.$ per group; $P<0.001$; Student's $t$ test; $\left.{ }^{* * *} P<0.001\right)$.

smaller than that recorded at $-40 \mathrm{mV}$ (Fig. 4e), indicating that there was an increase in the inward rectification of AMPARmediated currents. In recordings obtained from the shRNA group, the amplitude of AMPAR-eEPSCs evoked at $+40 \mathrm{mV}$ was equal to that of AMPAR-eEPSCs recorded at $-40 \mathrm{mV}$ (Fig. $4 \mathrm{f}$ ). The normalized I-V curves of AMPAR-eEPSCs also indicated obvious inward rectification at positive holding potentials in the Scr-seq group; in the shRNA group, however, the normalized I-V curves were linear, and no obvious inward rectification was detected at positive holding potentials (Fig. 4g). The RI of hippocampal CA1 neurons in the shRNA group was significantly lower than that of hippocampal CA1 neurons in the Scr-seq group (Fig. 4h). Based on these data, vezatin knockdown in the hippocampal CA1 region suppresses CP-AMPAR activity, indicating that vezatin may regulate AMPAR-mediated NST by influencing GluA2-lacking AMPARs.

\section{Vezatin modulates the surface expression of AMPAR GluA1 subunit (GluA1) by regulating the phosphorylation levels of GluA1 at serine $\mathbf{8 4 5}$}

We further assessed the effect of vezatin knockdown on the expression levels of two key AMPAR subunits, GluA1 and GluA2. Vezatin knockdown reduced the surface expression of GluA1 but not the expression level of total GluA1 in the hippocampi of epileptic mice (Fig. 5a, b). Vezatin knockdown did not alter the surface expression levels of GluA2 or the total expression of GluA2 in the hippocampi of epileptic mice (Fig. 5a, b). Moreover, in the in vitro seizure-like model, immunofluorescence staining indicated that vezatin knockdown decreased the surface expression level of GluA1 (Fig. 5c, d) but did not alter the total GluA1-expression level (Fig. 5e, f). These data indicate a role for vezatin in regulating the surface expression of GluA1 in vivo and in vitro. The major phosphorylation sites of GluA1 are serine 845 and serine 831; phosphorylation of these sites, phospho-GluA1 serine 845 (pGluA1-S845) and phospho-GluA1 serine 831 (pGluA1-S831), regulates the targeting of GluA1 to or its retention at the cell surface, further affecting the accumulation of surface GluA1 $[9,10]$. Thus, we further evaluated the effect of vezatin knockdown on levels of pGluA1-S845 and pGluA1-S831 in mice. Vezatin knockdown reduced the level of pGluA1-S845 but not pGluA1-S831 (Fig. 5a, b), suggesting that vezatin regulates the surface expression of GluA1 by influencing the levels of pGluA1-S845.

\section{Vezatin modulates the level of pGluA1-S845 by influencing protein kinase $A$ (PKA) activity}

The level of pGluA1-S845 is mainly determined by PKA- and calcium/calmodulin-dependent kinase II-a (CaMKII-a)-mediated phosphorylation of GluA1 at serine 845 and by protein phosphatase (PP) 2B-, PP2A-, and PP1-mediated dephosphorylation of GluA1 at serine $845[8,11]$. We postulated that vezatin plays a role in regulating the abovementioned signaling mechanisms, further affecting the level of pGluA1-S845. First, the coimmunoprecipitation analysis indicated that vezatin interacts with PKA (Fig. 6a) and CaMKII-a (Supplementary Fig. S6) but not PP2B, PP2A, or PP1 in the mouse hippocampus (Supplementary Fig. S6). Vezatin knockdown in the hippocampi of epileptic mice reduced the level of phosphoPKA (pPKA) (Fig. 6b, c) but did not alter the level of CaMKII-a phosphorylation (Supplementary Fig. S6) or the expression levels of PP2B (Fig. 6b, c), PP2A and PP1 (Supplementary Fig. S6). Based on these data, we speculated that vezatin plays a role in regulating the activation of PKA signaling, further affecting the level of pGluA1-S845. AKAP150 is known to anchor PKA when it targets pGluA1-S845 [8, 12], which may be involved in the regulation of PKA signaling by vezatin. Thus, we also evaluated the interaction between vezatin and AKAP150. The coimmunoprecipitation analysis indicated that vezatin interacts with AKAP150 (Supplementary Fig. S6); however, vezatin knockdown did not alter the expression of AKAP150 in the hippocampi of epileptic mice (Fig. 6b, c).

Next, we explored the underlying mechanism by which vezatin regulates PKA signaling in a vitro seizure-like model. Immunofluorescence staining showed that vezatin (purple) colocalized with PKA (blue) (Supplementary Fig. S7). Interestingly, vezatin knockdown reduced the colocalization of PKA and AKAP150 (Fig. 6d, e); moreover, vezatin knockdown promoted the internalization of PKA (blue) and AKAP150 (purple) in LV-infected neuronal dendrites (green) (Fig. 6d, f). These data suggest that vezatin knockdown inhibits the activation of PKA signaling and promotes the internalization of PKA and AKAP150 in epilepsy models.

To further confirm whether PKA mediates the effect of vezatin on the level of pGluA1-S845, H-89 (a selective inhibitor of PKA phosphorylation) was applied to inhibit the phosphorylation of PKA in cultured neurons in vitro. Western blot analysis showed that compared with the Scr-seq group that was not treated with $\mathrm{H}-89$, the $\mathrm{H}-89$-treated $\mathrm{Scr}$-seq group exhibited a decreased level of pPKA, indicating that $\mathrm{H}-89$ successfully suppressed the phosphorylation of PKA (Fig. $6 \mathrm{~g}, \mathrm{~h}$ ). No significant difference in the level of pPKA was observed between the Scr-seq group and shRNA group after treatment with $\mathrm{H}-89$ (Fig. 6h), indicating that $\mathrm{H}-89$ blocked the effect of vezatin knockdown on the phosphorylation of PKA. Furthermore, in the groups that was not treated with $\mathrm{H}-89$, vezatin knockdown reduced the level of pGluA1-S845 (Fig. 6i) and the surface expression of GluA1 (Fig. 6j); however, upon treatment with $\mathrm{H}-89$, no significant differences in the level of pGluA1-S845 (Fig. 6i) and the surface expression of GluA1 (Fig. 6j) were observed between the Scr-seq group and shRNA group. No significant difference in the total expression of GluA1 was observed among all the groups (Fig. 6k). These data indicate that $\mathrm{H}-89$ can block the downregulatory effect of vezatin knockdown on the expression of pGluA1-S845 and the surface expression of GluA1.

\section{Expression pattern of vezatin in subjects with epilepsy}

Vezatin expression in patients with temporal lobe epilepsy (TLE) was measured to further investigate the expression pattern of vezatin in epilepsy. Eleven controls with traumatic brain injury (TBI), i.e., six females and five males with a mean age of $29.00 \pm$ 3.70 years (mean \pm standard error), comprised the control group (Supplementary Table S1). Eleven patients with TLE, i.e., six females and five males with a mean age of $23.27 \pm 2.03$ years (mean \pm standard error), were included in the TLE group (Supplementary Table S2). There was no significant difference in age $(P=0.126)$ or sex $(P=1.000)$ between the two groups. Immunofluorescence staining showed that vezatin (green) was 


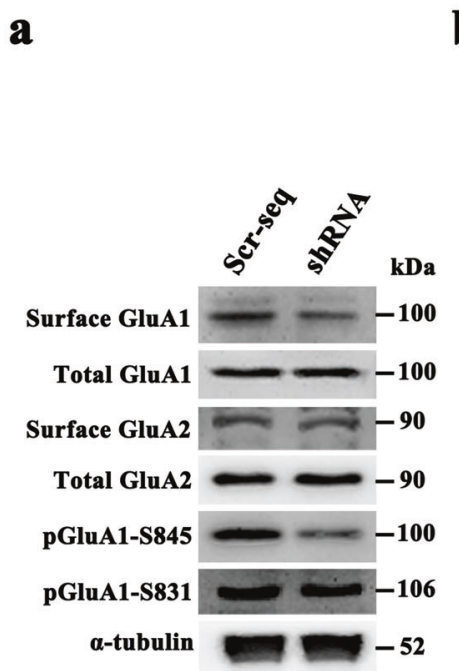

b
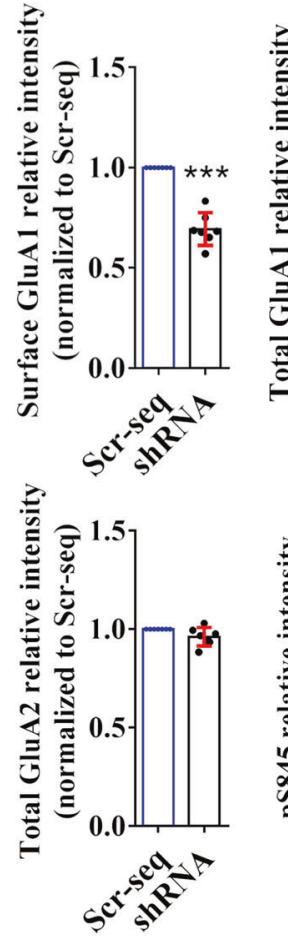

c
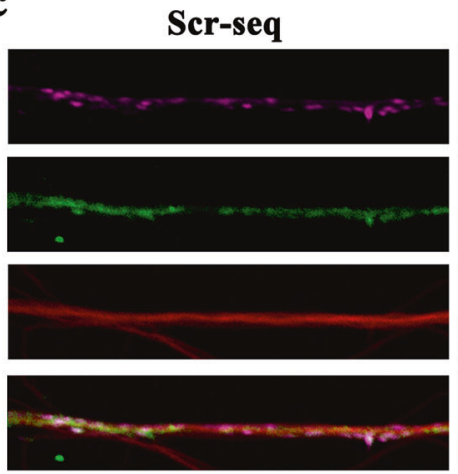

$\mathbf{e}$
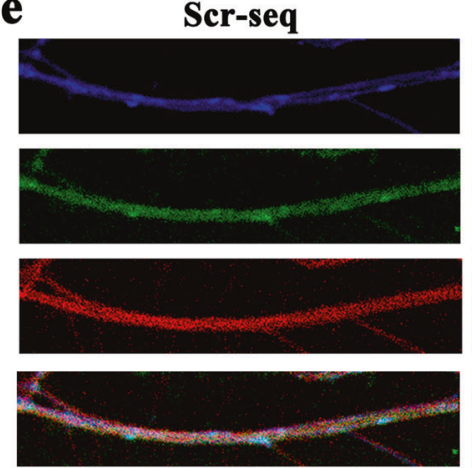
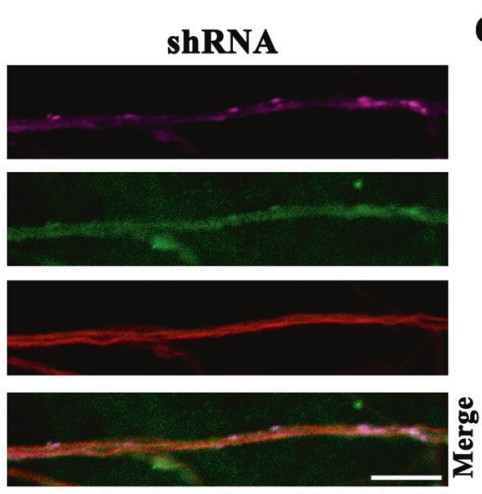

Surface GluA1/GFP/Tubb3
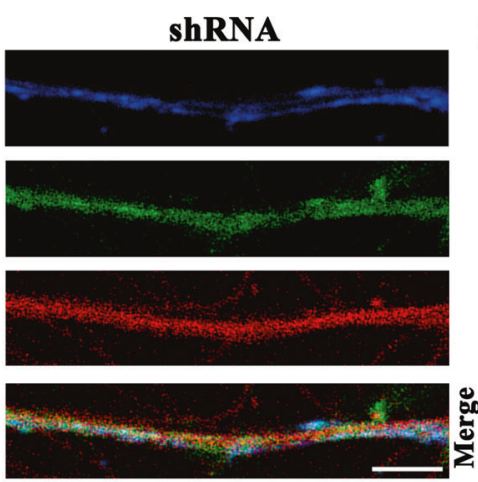

Total GluA1/GFP/Tubb3
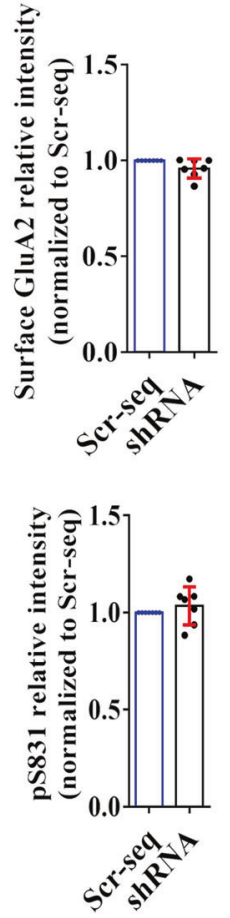

d

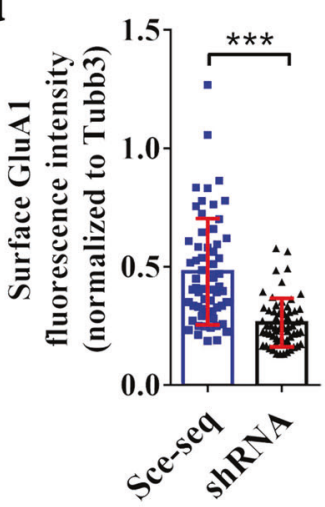

f

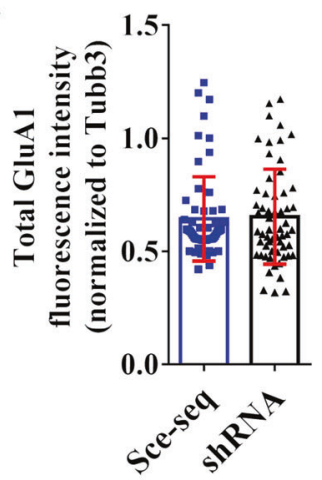

colocalized with the neuronal marker MAP2 (purple) in both the TLE group and the control group (Fig. 7a). Moreover, the fluorescence intensity of vezatin in the TLE group was higher than that in the control group (Fig. 7b). A subsequent western blot analysis suggested increased vezatin levels in the TLE group (Fig. 7c, d).

\section{DISCUSSION}

This study investigated the roles of vezatin in epilepsy. First, our study showed a significant increase in vezatin expression in the hippocampal tissues from epileptic mice and in an in vitro seizure-like model. Subsequent behavioral tests revealed that vezatin knockdown suppressed seizure activity in epileptic mice. 
Fig. 5 The effect of vezatin knockdown on the expression levels of GluA1, GluA2, pGluA1-S845, and pGluA1-S831. In the PILO-induced epilepsy model: a representative images of western blots showing surface GluA1, total GluA1, surface GluA2, total GluA2, pGluA1-S845, and pGluA1-S831 levels in the hippocampus in the Scr-seq group and shRNA group and $\mathbf{b}$ the corresponding statistical analyses $(n=7$ per group; surface GluA1, $P<0.001$; pGluA1-S845, $P<0.001)$. In the $\mathrm{Mg}^{2+}$-free solution-induced in vitro seizure-like model, $\mathbf{c}$ representative images of immunofluorescence staining showing that surface GluA1 (purple) was localized in Tubb3 (red)-positive and LV-infected neuronal dendrites (green) and (d) the corresponding statistical analysis of the fluorescence intensity of surface GluA1 in the Scr-seq group and shRNA group ( $P<$ 0.001). e Representative images of immunofluorescence staining showing that total GluA1 (blue) was localized in Tubb3 (red)-positive and LVinfected (green) neuronal dendrites and (f) the corresponding statistical analysis of the fluorescence intensity of total GluA1 expression in the Scr-seq group and shRNA group ( $n=5$ independent hippocampal neuron cultures from ten mice per group). Student's $t$ test; ${ }^{*} P<0.05$, ${ }^{*} P<$ 0.01 , and ${ }^{* * *} P<0.001$.

We further explored the underlying mechanism by which vezatin regulates seizure activity. Subsequent electrophysiological studies revealed that vezatin knockdown suppressed CP-AMPARmediated synaptic events and inhibited the surface expression of GluA1 in epileptic mice, indicating that vezatin may regulate AMPAR-mediated synaptic events by affecting the surface expression of GluA1. The accumulation of surface GluA1 is largely determined by the level of pGluA1-S845 [8]. Interestingly, vezatin knockdown decreased the level of pGluA1-S845; moreover, vezatin knockdown reduced the phosphorylation of PKA, an important regulator that promotes phosphorylation of GluA1 at serine $845[8,11]$. When the phosphorylation of PKA was suppressed by $\mathrm{H}-89$, the effects of vezatin knockdown on the phosphorylation of GluA1 at serine 845 and the surface expression of GluA1 were blocked, indicating that vezatin could modulate the level of pGluA1-S845, further regulating the surface expression of GluA1, which requires the activation of PKA signaling. Finally, we found that vezatin expression was increased in brain tissues from patients with TLE, which suggests a possible relationship between vezatin and epilepsy in humans.

Vezatin is a synaptic regulatory protein that can regulate NST $[3,4]$. In this study, vezatin expression was increased in the hippocampal CA1 region in epileptic mice, especially in the stratum radiatum. The hippocampal CA1 region contains many pyramidal neurons, which project dendrites and axons that form synapses with excitatory and inhibitory synaptic inputs mainly located in the stratum radiatum of the CA1 region $[2,13]$. Thus, the increased vezatin expression in the hippocampal CA1 region in epileptic mice revealed by our immunofluorescence analyses suggests a possible function for vezatin in regulating NST in epilepsy. Moreover, in vitro immunofluorescence staining indicated that vezatin was localized in the postsynaptic component of excitatory synapses, further suggesting that vezatin regulates seizure activity by affecting NST. Using the whole-cell patch-clamp technique, we determined that vezatin knockdown could decrease the amplitude of AMPAR-mEPSCs in epileptic mice, indicating that vezatin regulates seizure activity by affecting AMPAR-mediated NST. As key glutamate-gated ion channels located on the postsynaptic membrane, AMPARs mediate the majority of fast excitatory NST in the brain, which plays a vital role in the development of epilepsy [8]. CP-AMPARs, the majority of which are likely GluA1 homomers, are widely expressed in synapses during the early development of the brain $[8,11]$; with the development of the brain, CP-AMPARs are progressively lost in the majority of synapses on excitatory pyramidal neurons in the mature brain $[8,11]$. In the seizure model, however, CP-AMPARmediated synaptic events were significantly enhanced [14]. Thus, we postulated that vezatin plays a role in regulating CP-AMPARs that underlies epilepsy. Next, we found that vezatin knockdown could suppress CP-AMPAR-mediated synaptic events in the hippocampi of epileptic mice. Moreover, vezatin knockdown reduced the surface expression of GluA1 in vivo and in vitro. These data indicate that vezatin can affect AMPAR-mediated NST by regulating the surface expression of GluA1 in epilepsy.

Serine 845 is the major phosphorylation site of AMPARs, and phosphorylation at serine 845 largely affects the targeting of
AMPARs to or retention of AMPARs at the cell surface $[8,11]$. PKA and CaMKII promote the phosphorylation of GluA1 at serine 845, further facilitating the targeting of AMPARs to the cell surface and enhancing AMPAR-mediated NST $[8,11,12]$. In this study, vezatin interacted with PKA and CaMKII- $a$ by coimmunoprecipitation. Furthermore, vezatin knockdown inhibited the phosphorylation of PKA but had no effect on the phosphorylation of CaMKII-a in epileptic mice. These data indicate that PKA may mediate the role of vezatin in regulating the level of pGluA1-S845. The level of pGluA1-S845 is also affected by the expression of the proteins that dephosphorylated GluA1, including PP2B (which plays a major role), PP2A, and PP1 [11, 15]. Coimmunoprecipitation indicated negative interactions between vezatin and the three dephosphorylating proteins; accordingly, vezatin knockdown exerted no effect on the expression levels of these proteins. AKAP150 is known to anchor PKA when it targets pGluA1-S845 [12]; dysfunction of AKAP150 might affect the phosphorylation of GluA1 at serine 845 by PKA $[12,16]$. Thus, we postulated that there may be a relationship between vezatin and AKAP150. Coimmunoprecipitation revealed an interaction between vezatin and AKAP150; however, vezatin knockdown did not alter the expression of AKAP150 in epileptic mice. Interestingly, subsequent immunofluorescence analyses in the in vitro model indicated that vezatin knockdown decreased the colocalization of PKA with AKAP150, suggesting that vezatin knockdown may inhibit the binding of PKA to AKAP150. Moreover, immunofluorescence staining suggested that vezatin knockdown seemed to promote the internalization of both PKA and AKAP150 in neuronal dendrites in the in vitro seizure-like model, possibly influencing the effect of PKA and AKAP150 on targeting of GluA1 to the cell surface and subsequently affecting AMPAR-mediated synaptic activity. $\mathrm{H}-89$ was used to inhibit the phosphorylation of PKA in the in vitro seizure-like model and to further assess the mediating effect of PKA on the regulation of pGluA1-S845 by vezatin. When $\mathrm{H}-89$ was administered, the downregulatory effects of vezatin knockdown on the expression of pGluA1-S845 and the surface expression of GluA1 were inhibited, indicating that $\mathrm{H}-89$ blocked the effect of vezatin knockdown on the phosphorylation of GluA1 at serine 845, further inhibiting the effect of vezatin knockdown on the surface expression of GluA1. Based on these data, increased vezatin expression promotes PKA activation, further increasing the phosphorylation of GluA1 at serine 845, facilitating the targeting of AMPARs to the cell surface, and ultimately enhancing AMPAR-mediated NST and promoting seizure activity in epilepsy (Fig. 8).

A limitation of this study should be considered when interpreting the results. In the present study, cultured hippocampal neurons were exposed to $\mathrm{Mg}^{2+}$-free medium to mimic seizurelike activity in vitro and to explore the underlying mechanism of vezatin in epilepsy. Although this in vitro model has been routinely used to investigate the underlying molecular mechanisms of epilepsy, the cultured hippocampal neurons in vitro did not form anatomical connections or exhibit brain functions and did not display the clinical seizures observed in humans with epilepsy [17]. The PILO-induced mouse model mimicking acquired epilepsy also does not completely represent the complex epileptic 
$\mathbf{a}$

b

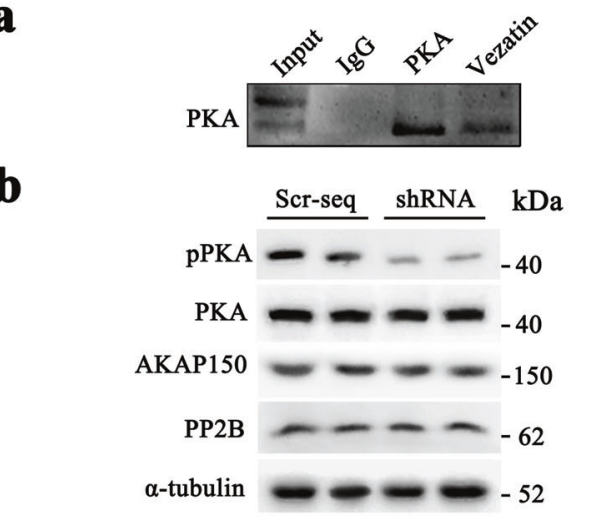

d
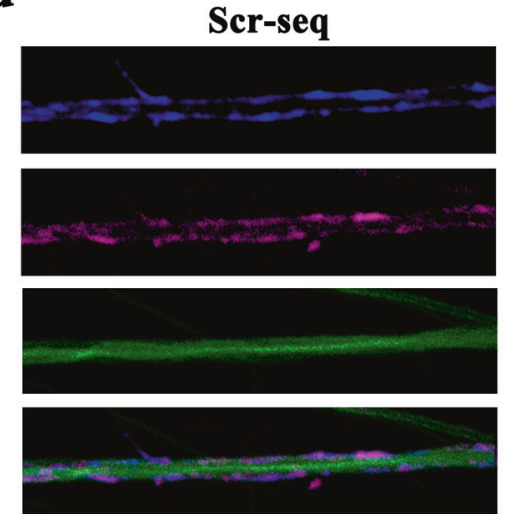

f

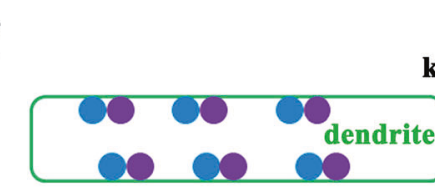

Vezatin

knockdown
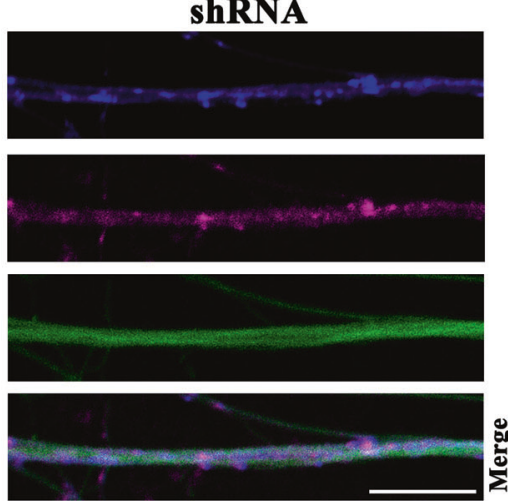

PKA/AKAP150/GFP
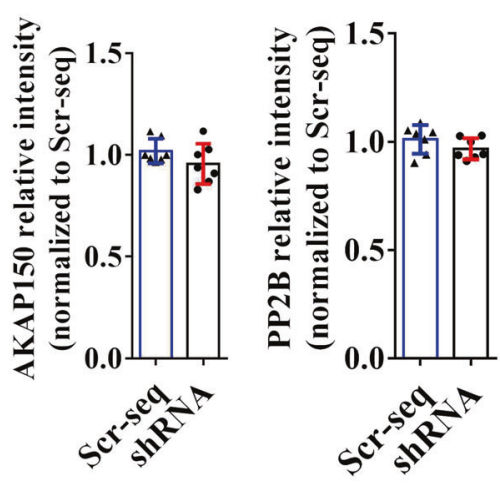

e

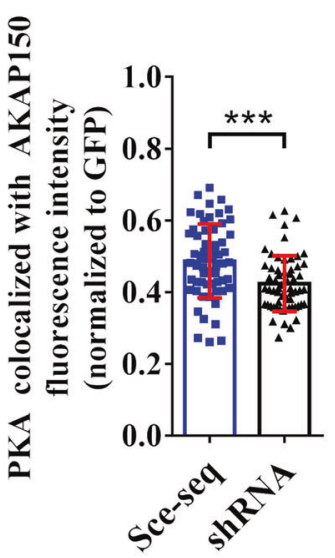

g

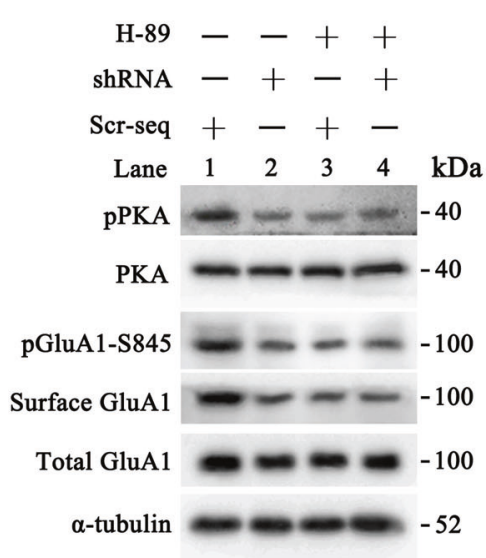

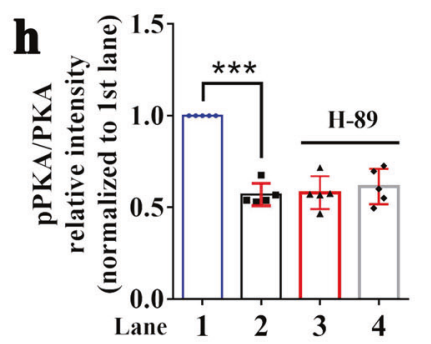

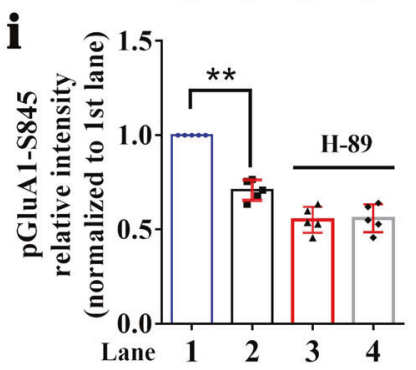

PKA

AKAP150

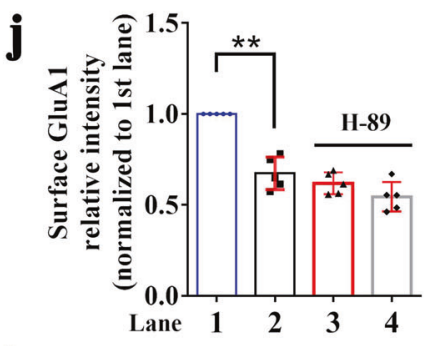

$\mathbf{k}$

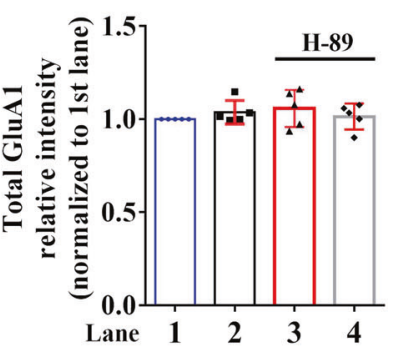

condition in humans with epilepsy [18]. Thus, our findings regarding the function and the molecular mechanism of vezatin in regulating seizure activity did not completely reflect the molecular mechanism of vezatin underlying the clinical epileptic condition. The function and the molecular mechanism of vezatin in human epilepsy remain undetermined.
In summary, we demonstrated that the expression pattern of vezatin is abnormal in epilepsy and that vezatin regulates seizure activity by affecting AMPAR-mediated NST and the surface expression of GluA1, which is involved in PKA-mediated phosphorylation of GluA1 at serine 845 . These findings may provide a novel target for seizure control. 
Fig. 6 PKA signaling mediates the regulatory effect of vezatin on the phosphorylation of GluA1 at serine 845 . a Representative coimmunoprecipitation image showing the interaction between vezatin and PKA in the hippocampi of mice. In the PILO-induced epilepsy model, $\mathbf{b}$ representative images of western blots showing pPKA levels, which was calculated as the pPKA to total PKA ratio, and the expression of AKAP150 and PP2B in the hippocampus of the Scr-seq group and shRNA group and $\mathbf{c}$ the corresponding statistical analyses ( $n=7$ per group; $\mathrm{PPKA} / \mathrm{PKA}, P<0.001$; Student's $t$ test; $\left.{ }^{* *} P<0.001\right)$. In the $\mathrm{Mg}^{2+}$-free medium-induced in vitro seizure-like model, $\mathbf{d}$ representative images of immunofluorescence staining comparing the distribution and colocalization (blue-purple) of PKA (blue) and AKAP150 (purple) in LV-infected neuronal dendrites (green) between the Scr-seq group and shRNA group and (e) the corresponding statistical analysis of the fluorescence intensity of the colocalization of PKA and AKAP150 between the Scr-seq group and shRNA group $(P<0.001$; Student's $t$ test; $\left.{ }^{* * *} P<0.001\right)(n=5$ independent hippocampal neuron cultures from ten mice per group). $f$ The corresponding schematic of the effect of vezatin knockdown on the distribution and colocalization of PKA and AKAP150 is also shown. In the Mg ${ }^{2+}$-free medium-induced in vitro seizure-like model, $\mathbf{g}$ representative images of western blots show that $\mathrm{H}-89$ blocks the effect of vezatin knockdown on the level of pGluA1S845 and the surface expression of GluA1, along with the corresponding statistical analyses of pPKA/PKA $(P<0.001)(\mathbf{h}), \mathrm{pGluA} 1-\mathrm{S} 845(P=$ $0.001)$ (i), surface GluA1 $(P=0.003)(j)$, and total GluA1 expression $(\mathbf{k})(n=5$ independent hippocampal neuron cultures from ten mice per group). Two-way ANOVA followed by the Bonferroni post hoc test; ${ }^{*} P<0.05,{ }^{* *} P<0.01$, and ${ }^{* * *} P<0.001$.

\section{MATERIALS AND METHODS}

\section{Animals}

All animal experiments performed in this study were approved by the Committee on Animal Research of Chongqing Medical University, Chongqing, China. Healthy adult male C57BL/6 mice (8-10 weeks old and $20-25 \mathrm{~g}$ ) were obtained from the Experimental Animal Center of Chongqing Medical University. All mice were raised in a temperaturecontrolled room $\left(24-26^{\circ} \mathrm{C}\right)$ on a $12 \mathrm{~h}$ light/dark cycle and provided free access to food and water.

\section{Mouse model of PILO-induced epilepsy and behavioral recordings}

Epilepsy was induced by PILO according to a method described in a previously published study [18]. PILO-induced seizures were classified according to Racine's standard criteria (stages 1-5) [19]. Before PILO administration, the mice were intraperitoneally (i.p.) injected with lithium chloride ( $\mathrm{LiCl}, 127 \mathrm{mg} / \mathrm{kg}$, Sigma-Aldrich, USA) for $20 \mathrm{~h}$. Atropine methyl nitrate $(1 \mathrm{mg} / \mathrm{kg})$ was i.p. injected $30 \mathrm{~min}$ before PILO administration to reduce the peripheral cholinomimetic effects of PILO. To induce status epilepticus (SE), PILO (50 mg/kg, Sigma-Aldrich, USA) was i.p. administered. Only mice that had persistent convulsive SE (stage 4 or 5 ) were considered for further study. Mice exhibiting convulsive SE for $1 \mathrm{~h}$ were i.p. injected with $10 \mathrm{mg} / \mathrm{kg}$ diazepam to terminate SE. After the acute SE stage, the SRSs of mice were monitored continuously with a video recording system for 30 consecutive days; SRSs were also classified according to Racine's criteria [19]. Only mice that exhibited SRSs were included in the epilepsy group. Hippocampal LFPs were also recorded to confirm the induction of PILOinduced epilepsy in mice. Mice in the control group were i.p. administered the same volume of $0.9 \%$ saline.

In addition to SE and SRSs, hippocampal LFPs were monitored to confirm the establishment of PILO-induced epilepsy in mice. The procedure for recording LFPs was described previously [20]. Briefly, mice were deeply anesthetized by i.p. injected sodium pentobarbital $(50 \mathrm{mg} / \mathrm{kg}$ ) and mounted on a stereotaxic apparatus (RWD Life Science, China). Electrodes were implanted in the dorsal hippocampal region (anterior-posterior, $2.0 \mathrm{~mm}$; medial-lateral, $1.5 \mathrm{~mm}$; dorsal-ventral, $1.5 \mathrm{~mm}$ ), and electrodes connected to a signal generator were fixed to the skull with dental acrylic cement. The MAP data acquisition system (Plexon, USA) was used to monitor and record LFPs. LFPs were further analyzed using Neuroexplorer software (Nex Technologies, USA). PILO-induced epileptiform discharges were recorded in the acute SE stage (Fig. 1a). Moreover, we recorded SEDs in vivo, named SLEs, after the acute induced SE stage (Fig. 1b); SLEs were defined as spontaneous paroxysmal polyspike discharges lasting for more than $5 \mathrm{~s}$ with a high amplitude ( $>2$ times the baseline value) and a frequency $>5 \mathrm{~Hz}[21,22]$.

\section{LV construction and stereotaxic injection}

An LV expressing a shRNA targeting vezatin was constructed to knock down vezatin expression in the hippocampal region. The LV expressed a transgene-encoding green fluorescent protein (GFP). The LV expressing the vezatin-targeting shRNA (AGCCAACTITCAAGCCGCAAGGCTA) was used to knock down the expression of vezatin in the shRNA group, and an LV expressing a scramble sequence (TTCTCCGAACGTGTCACGTAA) was used as a negative control in the Scr-seq group. All LVs were manufactured by Hanbio Biotechnology (Shanghai, China). The titer of these LVs was $5 \times 10^{8} \mathrm{TU} / \mathrm{ml}$.
The procedures for performing i.p. stereotaxic injection have been described previously $[20,23]$. Briefly, the mice were deeply anesthetized by an i.p. injection of sodium pentobarbital $(50 \mathrm{mg} / \mathrm{kg})$ and mounted on a stereotaxic apparatus. The lentivirus $(2 \mu \mathrm{l})$ was stereotactically injected into the hippocampal CA1 region (anterior-posterior, $2.0 \mathrm{~mm}$; medial-lateral, $1.3 \mathrm{~mm}$; dorsal-ventral, $1.4 \mathrm{~mm}$ ) at a speed of $0.4 \mu \mathrm{l} / \mathrm{min}$ with a $5-\mu \mathrm{l}$ syringe. The syringe was maintained in situ for an additional $5 \mathrm{~min}$ and then withdrawn slowly to prevent reflux.

To determine the effects of vezatin knockdown on seizure activity in the PILO-induced epilepsy model, mice were i.p. injected with PILO 2 weeks after hippocampal injection of LV. SRSs were monitored continuously with a video-monitoring system ( $24 \mathrm{~h} /$ day) for 30 consecutive days. We also monitored and recorded hippocampal LFPs. The latency period, frequency, and Racine stage of SRSs and number of SLEs were determined and analyzed independently by two researchers. The SRS latency was defined as the interval between PILO injection and the onset of the first SRS.

\section{Primary neuron culture and LV transfection}

Hippocampal tissues were dissected from postnatal (P0-1) C57BL/6 mice. Hippocampal explants were digested with trypsin and then mechanically triturated. Next, a diluted cell suspension was plated in poly-L-lysine-coated dishes, and the neurons were incubated in a cell culture incubator at $37^{\circ} \mathrm{C}$ for $4 \mathrm{~h}$. For hours after plating, the medium was replaced with neurobasal medium supplemented with 2\% B27 (Gibco, USA), 1\% antibiotics, and $0.5 \mathrm{mM}$ L-glutamine in a humidified incubator at $37^{\circ} \mathrm{C}$ [24]. Then, $\sim 1 / 2$ of the culture medium was changed every 3 days.

Cultured hippocampal neurons that display SEDs have been well characterized as a useful in vitro model of seizures [17, 24]. We generated this in vitro seizure model by replacing the culture medium with a $\mathrm{Mg}^{2+}$ free extracellular solution (in $\mathrm{mM})\left(145 \mathrm{NaCl}, 10 \mathrm{HEPES}, 2.5 \mathrm{KCl}, 2 \mathrm{CaCl}_{2}, 10\right.$ glucose, 0.002 glycine; $\mathrm{pH} 7.3$; osmolarity adjusted to $320 \mathrm{mOsm}$ ) for $3 \mathrm{~h}$ on DIV 14. In the control group, the culture medium was replaced with extracellular solution containing $1 \mathrm{mM} \mathrm{MgCl}$. Then, in both groups, the extracellular solution was replaced with fresh culture media. After $24 \mathrm{~h}$, the SEDs of cultured neurons were recorded using the whole-cell patch-clamp technique to confirm the establishment of the in vitro seizure-like model (Supplementary Fig. S1). An SED was defined as a spontaneous highfrequency burst of action potentials (APs) ( $\geq 4$ APs) $[25,26]$.

To knockdown the expression of vezatin in vitro, cultured neurons in the shRNA group were transfected with an LV expressing shRNA targeting vezatin on DIV 3. Neurons in the Scr-seq group were transfected with an LV expressing a scramble sequence as a negative control. To determine the effects of vezatin knockdown on SEDs in vitro, transfected neurons were exposed to $\mathrm{Mg}^{2+}$-free extracellular solution on DIV14 [24]. After $24 \mathrm{~h}$, the SEDs were recorded using the whole-cell patch-clamp technique.

Cultured neurons were incubated with medium containing $30 \mu \mathrm{M} \mathrm{H}-89$ (MedChemExpress) for $2 \mathrm{~h}$ to inhibit the phosphorylation of PKA in vitro [27] and then with $\mathrm{Mg}^{2+}$-free extracellular solution. Then, in both groups, the extracellular solution was replaced with fresh culture media. After $24 \mathrm{~h}$, neurons were harvested for western blot analysis.

\section{Subjects with epilepsy and controls}

The human study complied with the Declaration of Helsinki and the ethical principles of the National Institutes of Health and was approved by the Committee on Human Research of the Second Affiliated Hospital of Chongqing Medical University. All human brain tissue samples were obtained as described in our previous studies [20,28]. Brain tissue samples 

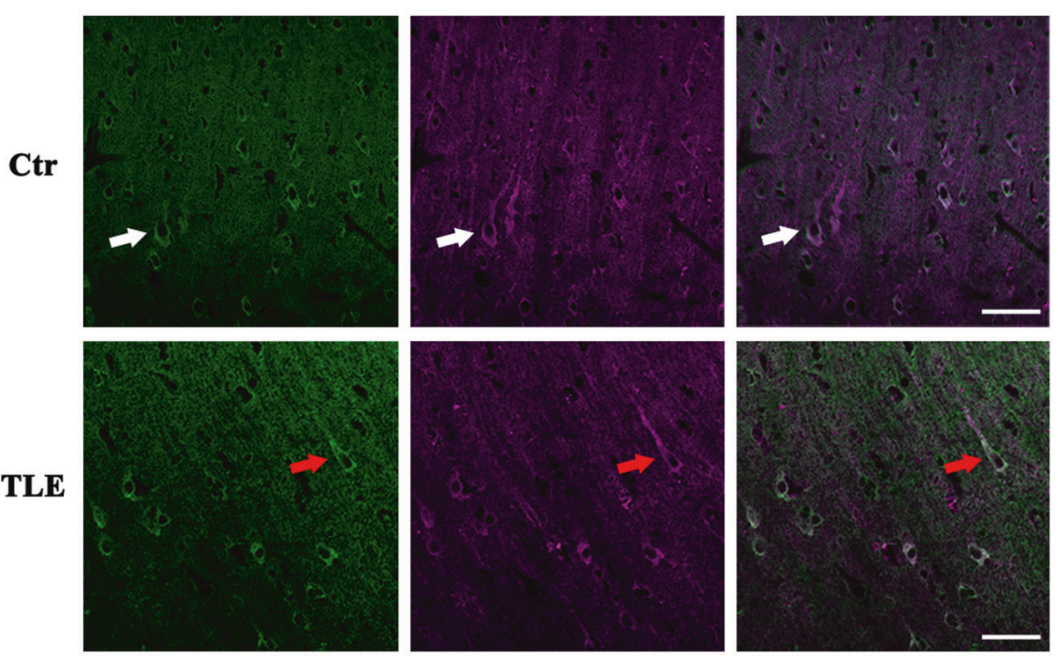

b

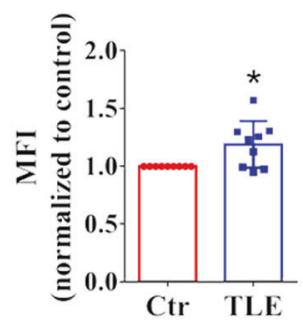

c d

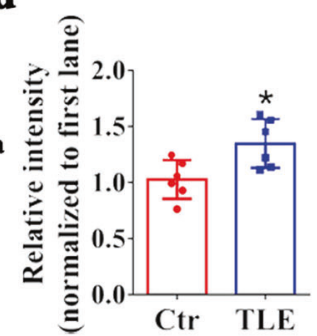

Fig. 7 The pattern of vezatin expression in subjects with epilepsy. a Representative images of immunofluorescence staining showing that in both the control group (white arrow) and TLE group (red arrow), vezatin (green) colocalized with the neuronal marker MAP2 (purple), and b the corresponding statistical analyses of the MFI of vezatin $(n=9$ per group, $P=0.023)$. c Representative images of western blots showing vezatin expression in the control group and TLE group and (d) the corresponding statistical analysis $(n=6$ per group, $P=0.017)$. Student's $t$ test; ${ }^{*} P<0.05,{ }^{* *} P<0.01$, and ${ }^{* * *} P<0.001$.

were obtained from 11 patients undergoing surgery for medically refractory TLE and 11 controls undergoing surgery for TBI at the Xinqiao Hospital of the Third Military University and the First Affiliated Hospital of Chongqing Medical University, China. Informed consent for the use of brain tissues in this study was obtained from the patients or their lineal relatives. Refractory TLE was diagnosed according to criteria proposed by the International League Against Epilepsy [29]. All patients with refractory TLE had typical symptoms and electroencephalogram (EEG) features, and their seizures were refractory to combination therapy with the maximal tolerable doses of at least three antiepileptic drugs (AEDs) for more than 2 years. The patients with refractory TLE underwent detailed medical evaluations, including assessment of epilepsy manifestations, a neurological examination, neuroimaging (e.g., positron emission tomographycomputed tomography or high-resolution magnetic resonance imaging), and electrophysiological examination ( $24 \mathrm{~h}$ video EEG), prior to surgery. The epileptic focus was also localized during surgery via intraoperative EEG. Brain tissue samples were obtained from control group patients, i.e., patients who required craniocerebral surgery for increased intracranial pressure resulting from severe TBI and who had no history of epilepsy, seizures, or exposure to AEDs. The clinical data of these subjects are provided in Supplementary Tables S1 and S2.

\section{Immunofluorescence staining}

The immunofluorescence procedures were described previously $[20,28]$. The mice were rapidly euthanized, and mouse brain tissues were fixed with $4 \%$ paraformaldehyde for $24 \mathrm{~h}$; the human brain tissues were also fixed with $4 \%$ paraformaldehyde for $24 \mathrm{~h}$. The mouse brain tissues and human brain tissues were sequentially incubated in $20 \%$ and $30 \%$ graded sucrose solutions for $24 \mathrm{~h}$ and sliced into $15-\mu \mathrm{m}$ frozen sections using a freezing microtome. Then, the sections were collected on glass slides. The frozen sections were air-dried at room temperature for $10 \mathrm{~min}$ and immersed in acetone for $20 \mathrm{~min}$. Next, the sections were washed with phosphate-buffered saline (PBS) and permeabilized with $0.4 \%$ Triton
X-100. After that, the sections were blocked with $4 \%$ goat serum for $120 \mathrm{~min}$. For immunofluorescence analysis of cultured neurons, the neurons were plated and then allowed to adhere to coverslips; neurons on coverslips were fixed with $4 \%$ paraformaldehyde for $10 \mathrm{~min}$ and then permeabilized with $0.4 \%$ Triton X-100 and blocked with $4 \%$ goat serum. The sections and cultured neurons were incubated with primary antibodies overnight at $4{ }^{\circ} \mathrm{C}$. The following primary antibodies were used: a mouse vezatin antibody (Santa Cruz Biotechnology; 1:100), rabbit MAP2 antibody (Proteintech; 1:200), guinea pig Tubb3 antibody (Synaptic Systems; 1:200), mouse Tubb3 antibody (Proteintech; 1:400), rabbit PSD95 antibody (Abcam; 1:200), rabbit Synapsin 1 antibody (Novus; 1:200), rabbit GluA1 antibody (Abcam; 1:200), rabbit PKA antibody (Cell Signaling Technology; 1:100), and mouse AKAP150 antibody (Santa Cruz Biotechnology; 1:100). The following secondary antibodies were used: fluorescein isothiocyanate (FITC)-labeled goat anti-mouse IgG (Proteintech; 1:100), Alexa Fluor 555-labeled goat anti-rabbit IgG (Beyotime; 1:500), Alexa Fluor 647-labeled goat anti-rabbit IgG (Beyotime; 1:200), Alexa Fluor 555labeled goat anti-guinea pig IgG (Abcam; 1:500), Alexa Fluor 647-labeled goat anti-mouse IgG (Beyotime; 1:200), and AMCA-labeled goat antirabbit IgG (Proteintech; 1:200). Next, the sections and neurons on coverslips were extensively washed with PBS three times $(10 \mathrm{~min}$ per wash) and mounted with 50\% glycerol/PBS.

To label surface GluA1 on cultured neurons, neurons on coverslips were first fixed in 4\% paraformaldehyde and blocked in 4\% goat serum (without permeabilization) [30]. Then, the neurons were incubated with a mouse GluA1 N-terminus antibody (Millipore Sigma; 1:200) for $1 \mathrm{~h}$ in PBS and washed three times before being permeabilized [30]. After that, the neurons were permeabilized with $0.4 \%$ Triton X-100 and incubated with a Tubb3 antibody overnight at $4{ }^{\circ} \mathrm{C}$ for immunostaining as described above.

Fluorescence intensity was analyzed as described previously [20]. Fluorescence images were captured with a laser scanning confocal $\mathrm{Ti}$ microscope (Nikon, Japan). Images of brain tissues were captured using $\times 20$ objective (numerical aperture: 1.4; working distance: $1.0 \mathrm{~mm}$, corrected for $0.17 \mathrm{~mm}$ coverslips) with an acquisition setting of a $512 \times$ 


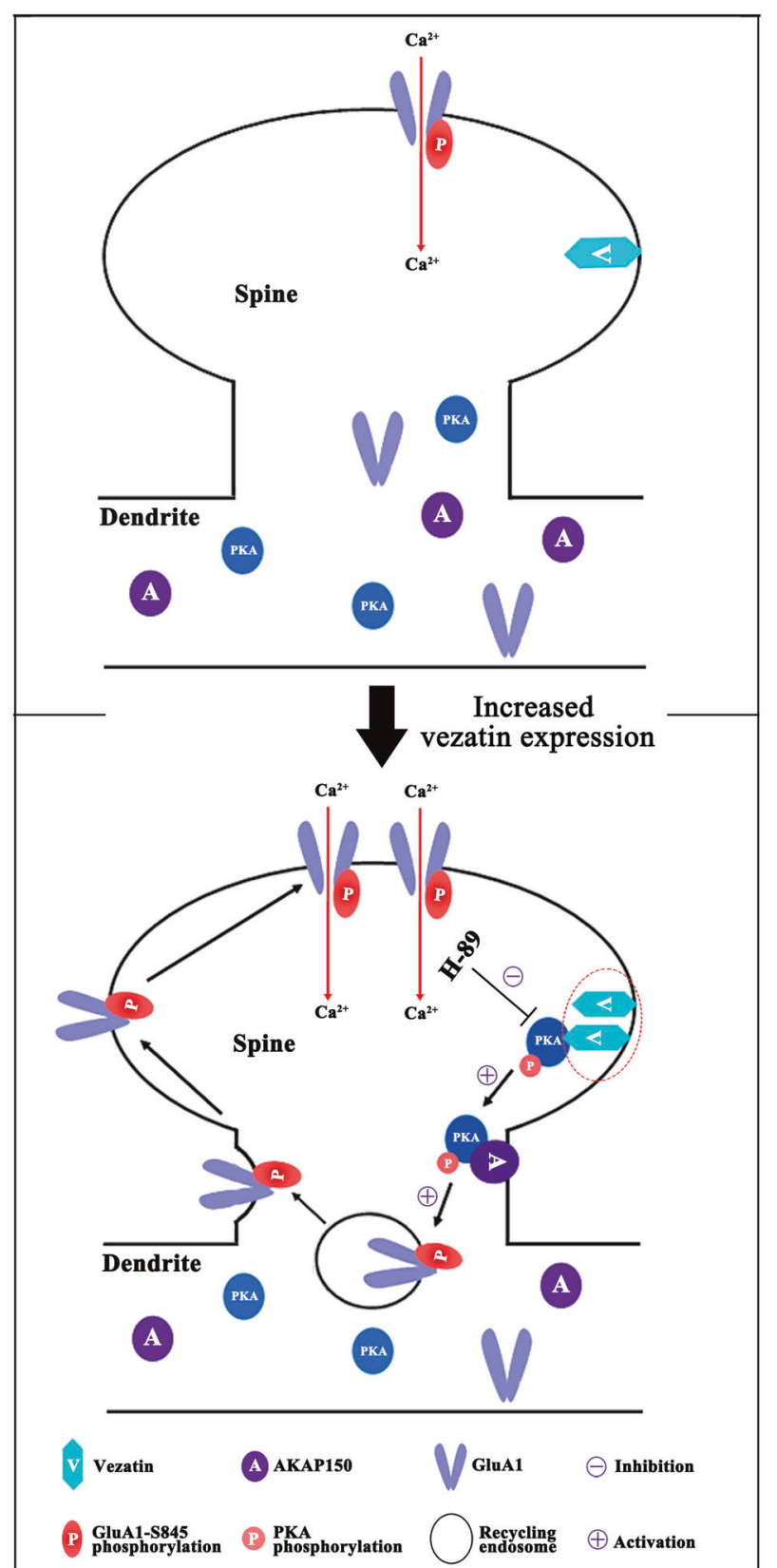

Fig. 8 Schematic of the mechanism by which vezatin regulates seizure activity. Increased vezatin expression promotes PKA activation (which can be blocked by $\mathrm{H}-89$ ), facilitating the targeting of pGluA1-S845 by PKA and AKAP150, further increasing the phosphorylation of GluA1 at serine 845 and promoting the targeting of AMPARs to the neuronal surface and then to postsynaptic elements, ultimately enhancing AMPAR-mediated synaptic transmission and promoting seizure activity in individuals with epilepsy.

512 pixels resolution. Images of cultured neurons were obtained with a $60 \mathrm{x}$ oil immersion objective (numerical aperture: 1.4; working distance $0.13 \mathrm{~mm}$, corrected for $0.17 \mathrm{~mm}$ coverslips) as z-series of $\sim 6-8$ images captured at $1 \mu \mathrm{m}$ intervals with an acquisition setting of a $1024 \times 1024$ pixels resolution.

The quantitative analysis of immunofluorescence staining was evaluated using Image-Pro Plus 6.0 software (Media Cybernetics). Images of vezatin immunofluorescence staining in the hippocampal CA1 region were corrected for the background and segmented to isolate specific vezatin staining from nonspecific fluorescence. The hippocampal CA1 region was divided into three layers: stratum oriens, pyramidal cell layer, and stratum radiatum [13]. For each layer obtained from hippocampal CA1 region of each mouse, the MFI (calculated as integrated fluorescence intensity divided by the region of interest (ROI)) of vezatin was measured over four separate rectangular ROIs to obtain an average of MFI values. All settings and measurement parameters (immunofluorescence excitation with emission, exposure duration, background correction, and size and dimensions of ROIs) for both control and epilepsy groups were maintained at constant values throughout. A MFI value for each acquired field was then normalized to the value of the control group. The quantitative analysis of immunofluorescence staining for vezatin in human brain tissues was similar to that used for vezatin in mouse brain tissues. For cultured neurons, fluorescence images were also corrected for background and segmented to isolate the staining for specific proteins of interest from nonspecific fluorescence. The fluorescence intensity of proteins of interest was measured over three separate ROls in each cell to obtain an average of intensity values. Intensities of GluA1 and vezatin were normalized to Tubb3 in the same ROl; the intensity of the colocalization (blue-purple) of PKA and AKAP150 was normalized to GFP in the same ROI. For each group, 12 cells were imaged and assessed per independent hippocampal neuron culture.

\section{Western blot analysis and coimmunoprecipitation}

The western blot procedures were described previously $[20,24,28]$. We extracted total protein from mouse brain tissues, human brain tissues, and cultured hippocampal neurons using a whole protein extraction kit (Beyotime). We isolated surface protein from mouse brain tissues and cultured hippocampal neurons using the Mem-PER Plus Membrane Protein Extraction Kit (Thermo Fisher Scientific, USA) according to the instructions for transmembrane protein extraction. Next, the protein samples $(50 \mu \mathrm{g}$ per lane) were separated by SDS-PAGE on a $5 \%$ stacking gel and a $10 \%$ separating gel and then transferred to a PVDF membrane (Merck Millipore, Germany). After that, the PVDF membranes were blocked at $37^{\circ} \mathrm{C}$ for $120 \mathrm{~min}$ in $5 \%$ skim milk and then incubated with primary antibodies overnight at $4{ }^{\circ} \mathrm{C}$. The following primary antibodies were used: a mouse vezatin antibody (Santa Cruz Biotechnology; 1:100), mouse GAPDH antibody (Proteintech; 1:5000), rabbit GluA1 antibody (Abcam; 1:200), rabbit GluA2 antibody (Proteintech; 1:500), rabbit pGluA1-S845 antibody (Abcam; 1:1000), rabbit pGluA1-S831 antibody (Abcam; 1:1000), rabbit atubulin antibody (Proteintech; 1:1000), rabbit PPKA (phospho-PKA threonine 197) antibody (Cell Signaling Technology; 1:1000), rabbit PKA antibody (Cell Signaling Technology; 1:1000), mouse AKAP150 antibody (Santa Cruz Biotechnology; 1:200), mouse PP1 antibody (Santa Cruz Biotechnology; 1:200), rabbit PP2A antibody (Abcam; 1:2000), mouse PP2B antibody (Santa Cruz Biotechnology; 1:200), rabbit CaMKIl-a antibody (Proteintech; 1:1000), rabbit phospho-CaMKIl-a (threonine 286) (pCaMKII-a) antibody (Cell Signaling Technology; 1:1000), and mouse $\beta$-actin antibody (Proteintech; 1:5000). Then, the membranes were washed with TBST and incubated with a horseradish peroxidase-conjugated goat anti-rabbit lgG antibody (Proteintech; 1:2000) and goat anti-mouse IgG antibody (Proteintech; 1:2000) for $1 \mathrm{~h}$ at $37^{\circ} \mathrm{C}$. After the membranes were washed with TBST, the protein bands were visualized using enhanced chemiluminescence (ECL) reagent (Beyotime) and a Fusion FX5 image analysis system (Vilber Lourmat). The density of each band was evaluated using a Fusion FX5 image analysis system and then normalized to that of the loading control for further analysis.

For coimmunoprecipitation, the protein was extracted from mouse brain tissues using a whole protein extraction kit and then incubated with $40 \mu \mathrm{l}$ of Protein $A+G$ agarose beads (Beyotime) with IgG antibody (Beyotime; mouse and rabbit) for $2 \mathrm{~h}$ at $4{ }^{\circ} \mathrm{C}$ before centrifugation at $1000 \times \mathrm{g}$ for $5 \mathrm{~min}$. The supernatants were collected and incubated with mouse vezatin antibody (Santa Cruz Biotechnology), rabbit PKA antibody (Cell Signaling Technology), mouse AKAP150 antibody (Santa Cruz Biotechnology), mouse PP1 antibody (Santa Cruz Biotechnology), rabbit PP2A antibody (Abcam), mouse PP2B antibody (Santa Cruz Biotechnology), rabbit CaMKII-a antibody (Proteintech), or lgG antibody (Beyotime; mouse and rabbit) overnight at $4{ }^{\circ} \mathrm{C}$, and then $40 \mu \mathrm{l}$ of Protein $\mathrm{A}+\mathrm{G}$ agarose beads were added and incubated for $3 \mathrm{~h}$ at $4{ }^{\circ} \mathrm{C}$. The immunoprecipitates were collected and washed with lysis buffer five times after centrifugation at $1000 \times g$ for $5 \mathrm{~min}$ and then analyzed by western blotting.

\section{In vitro electrophysiological recordings}

Whole-cell patch-clamp recordings were performed as described previously [20]. Briefly, mice were deeply anesthetized by i.p. injected sodium pentobarbital $(50 \mathrm{mg} / \mathrm{kg})$. The brains were rapidly removed from the mice, 
and $300-\mu \mathrm{m}$-thick coronal brain slices containing the hippocampus were cut with a vibratome (Leica, Germany) in ice-cold $\left(0-4^{\circ} \mathrm{C}\right)$ cutting solution (in mM) $\left(2.7 \mathrm{KCl}, 7.0 \mathrm{MgCl}_{2}, 0.5 \mathrm{CaCl}_{2}, 75.1\right.$ sucrose, $1.4 \mathrm{NaH}_{2} \mathrm{PO}_{4}, 26$ $\mathrm{NaHCO}_{3}, 25$ glucose, and $87.3 \mathrm{NaCl} ; \mathrm{pH}$ 7.35) that was continuously bubbled with carbogen $\left(95 \% \mathrm{O}_{2} / 5 \% \mathrm{CO}_{2}\right)$. Then, fresh brain slices were transferred to an incubation chamber containing artificial cerebral spinal fluid (ACSF) (in mM) $\left(125 \mathrm{NaCl}, 2.5 \mathrm{KCl}, 2.0 \mathrm{CaCl}_{2}, 1.25 \mathrm{NaH}_{2} \mathrm{PO}_{4}, 25\right.$ $\mathrm{NaHCO}_{3}, 10$ glucose, and $1.0 \mathrm{MgCl}_{2}$ ) and incubated at $34^{\circ} \mathrm{C}$ for $60 \mathrm{~min}$; the chamber was also continuously bubbled with carbogen $\left(95 \% \mathrm{O}_{2} / 5 \% \mathrm{CO}_{2}\right)$.

Hippocampal CA1 neurons were observed under an inverted phasecontrast microscope and chosen for whole-cell patch-clamp recordings. Excitatory synaptic transmission is mainly mediated by two types of ionotropic glutamate receptors: AMPARs and NMDARs [31, 32]. We, therefore, measured the AMPAR-mediated and NMDAR-mediated NST of hippocampal CA1 neurons. For mEPSC recordings, pipettes (3-8-M $\Omega$ polished glass pipettes) were filled with an internal solution (in $\mathrm{mM}$ ) (130 $\mathrm{CsMeSO}_{4}, 10 \mathrm{CsCl}_{2}, 10$ HEPES, $4 \mathrm{NaCl}, 1 \mathrm{MgCl}_{2}, 1$ EGTA, $5 \mathrm{MgATP}, 12$ phosphocreatine, $5 \mathrm{~N}$-methyl-D-glucamine (NMG), and $0.5 \mathrm{Na}_{3} \mathrm{GTP} ; \mathrm{pH}$ 7.20). AMPAR-mEPSCs were recorded in the presence of $1 \mu \mathrm{M}$ tetrodotoxin (TTX), $100 \mu \mathrm{M}$ picrotoxin (PTX), and $50 \mu \mathrm{M}$ 2-amino-5-phosphonovaleric acid (APV) at a holding potential of $-70 \mathrm{mV}$ in extracellular solution. NMDAR-mEPSCs were recorded in the presence of $1 \mu \mathrm{M} \mathrm{TTX}, 100 \mu \mathrm{M}$ PTX, and $20 \mu \mathrm{M}$ DNQX at a holding potential of $-60 \mathrm{mV}$ in extracellular solution. We also measured the inhibitory synaptic transmissions of hippocampal CA1 neurons: the mIPSCs of CA 1 neurons were recorded in ACSF in the presence of $1 \mu \mathrm{M} \mathrm{TTX,}$ $20 \mu \mathrm{M}$ DNQX, and $50 \mu \mathrm{M}$ APV at a holding potential of $-70 \mathrm{mV}$.

Next, the AMPAR-eEPSCs of hippocampal CA1 neurons were recorded. AMPAR-eEPSCs were recorded by stimulating Schaffer collaterals projecting to the CA1 region using a concentric bipolar tungsten stimulating electrode with settings of $0.05 \mathrm{~Hz}$ and $0.1 \mathrm{~ms}$ duration. The glass pipettes $(3-8 \mathrm{M} \Omega)$ of the recording electrode were filled with the same internal solution as described above for mEPSC recordings. AMPAReEPSCs were recorded in the presence of $100 \mu \mathrm{M}$ PTX and $50 \mu \mathrm{M}$ APV in extracellular solution. The current-voltage relationship (I-V curve) of the AMPAR-eEPSCs was obtained by determining the average amplitude of ten eEPSC peaks at various holding potentials $(-60$ to $+40 \mathrm{mV}$ ) $[14,33,34]$. The rectification index (RI) was calculated as the ratio of AMPAR-eEPSC amplitude at $-60 \mathrm{mV}$ to AMPAR-eEPSC amplitude at $+40 \mathrm{mV}\left(\mathrm{eEPSCs}_{-60 \mathrm{mV} / \mathrm{eEPSC}}+40 \mathrm{mv}\right)[33,34]$.

To record the APs of cultured hippocampal neurons in vitro, fresh hippocampal cultures were mounted on the stage and observed under an inverted phase-contrast microscope. The whole-cell patch-clamp recording was conducted to record APs at the resting membrane potential in current-clamp mode $[25,35]$. The pipettes $(3-5-\mathrm{M} \Omega$ polished glass pipettes) were filled with the following internal solution (in $\mathrm{mM}$ ): 17.5 $\mathrm{KCl}, 0.5 \mathrm{EGTA}, 122.5$ potassium gluconate, $10 \mathrm{HEPES}$, and $4 \mathrm{Na}_{2} \mathrm{ATP} ; \mathrm{pH}$ 7.2. All AP recordings were conducted with settings at $I=0$ and no current was injected in AP recordings.

To monitor and acquire electrophysiological data, a MultiClamp 700B amplifier (Axon, USA) and a Digidata $1322 \mathrm{~A}$ interface (Axon, USA) were used. Then, the data were recorded and analyzed with PCLAMP 9.2 software (Molecular Devices, USA).

\section{Statistical analysis}

Prior to analysis, all datasets were tested for normality and homogeneity of variance using the Kolmogorov-Smirnov test and Levene's test, respectively. Normally distributed and homogeneous data are presented as the means \pm standard errors. Comparisons between two groups were performed using unpaired Student's two-tailed $t$ test. Comparisons between multiple groups (more than two groups) considering one fixed factor were performed using one-way analysis of variance (ANOVA) followed by the Bonferroni post hoc test, and comparisons considering two fixed factors were performed using two-way ANOVA followed by the Bonferroni post hoc test. Nonnormally distributed or nonhomogeneous data are presented as the median and range, and the nonparametric Kruskal-Wallis test was used for statistical analyses. Fisher's exact test was performed for the comparison of sex differences between patients with TLE and control subjects. Statistical significance was set at $P<0.05$. SPSS 20.0 and GraphPad Prism 5.0 software were used for statistical analyses and to generate graphs, respectively.

\section{DATA AVAILABILITY}

The datasets generated and analyzed during this study are available from the corresponding author on reasonable request.

\section{REFERENCES}

1. Moshe SL, Perucca E, Ryvlin P, Tomson T. Epilepsy: new advances. Lancet. 2015;385:884-98.

2. Goldberg EM, Coulter DA. Mechanisms of epileptogenesis: a convergence on neural circuit dysfunction. Nat Rev Neurosci. 2013;14:337-49.

3. Danglot L, Freret T, Le Roux N, Narboux Neme N, Burgo A, Hyenne V, et al. Vezatin is essential for dendritic spine morphogenesis and functional synaptic maturation. J Neurosci. 2012;32:9007-22.

4. Sanda M, Ohara N, Kamata A, Hara Y, Tamaki H, Sukegawa J, et al. Vezatin, a potential target for ADP-ribosylation factor 6 , regulates the dendritic formation of hippocampal neurons. Neurosci Res. 2010;67:126-36.

5. Bahloul A, Simmler MC, Michel V, Leibovici M, Perfettini I, Roux I, et al. Vezatin, an integral membrane protein of adherens junctions, is required for the sound resilience of cochlear hair cells. EMBO Mol Med. 2009;1:125-38.

6. Koppel N, Friese MB, Cardasis HL, Neubert TA, Burden SJ. Vezatin is required for the maturation of the neuromuscular synapse. Mol Biol Cell. 2019;30:2571-83.

7. Navidhamidi M, Ghasemi M, Mehranfard N. Epilepsy-associated alterations in hippocampal excitability. Rev Neurosci. 2017;28:307-34.

8. Diering GH, Huganir RL. The AMPA receptor code of synaptic plasticity. Neuron. 2018;100:314-29.

9. Man HY. GluA2-lacking, calcium-permeable AMPA receptors-inducers of plasticity? Curr Opin Neurobiol. 2011;21:291-8.

10. He K, Song L, Cummings LW, Goldman J, Huganir RL, Lee HK. Stabilization of Ca2 +-permeable AMPA receptors at perisynaptic sites by GluR1-S845 phosphorylation. Proc Natl Acad Sci USA. 2009;106:20033-8.

11. Sanderson JL, Dell'Acqua ML. AKAP signaling complexes in regulation of excitatory synaptic plasticity. Neuroscientist. 2011;17:321-36.

12. Diering GH, Gustina AS, Huganir RL. PKA-GluA1 coupling via AKAP5 controls AMPA receptor phosphorylation and cell-surface targeting during bidirectional homeostatic plasticity. Neuron. 2014;84:790-805.

13. Piskorowski RA, Chevaleyre V. Synaptic integration by different dendritic compartments of hippocampal CA1 and CA2 pyramidal neurons. Cell Mol Life Sci. 2012;69:75-88

14. Rajasekaran K, Todorovic M, Kapur J. Calcium-permeable AMPA receptors are expressed in a rodent model of status epilepticus. Ann Neurol. 2012;72:91-102.

15. Hell JW. How Ca2+-permeable AMPA receptors, the kinase PKA, and the phosphatase PP2B are intertwined in synaptic LTP and LTD. Sci Signal. 2016;9:e2.

16. Purkey AM, Woolfrey KM, Crosby KC, Stich DG, Chick WS, Aoto J, et al. AKAP150 palmitoylation regulates synaptic incorporation of $\mathrm{Ca}(2+)$-permeable AMPA receptors to control LTP. Cell Rep. 2018;25:974-87 e4.

17. Deshpande LS, Nagarkatti N, Ziobro JM, Sombati S, DeLorenzo RJ. Carisbamate prevents the development and expression of spontaneous recurrent epileptiform discharges and is neuroprotective in cultured hippocampal neurons. Epilepsia. 2008;49:1795-802.

18. Curia G, Longo D, Biagini G, Jones RS, Avoli M. The pilocarpine model of temporal lobe epilepsy. J Neurosci Methods. 2008;172:143-57.

19. Racine RJ. Modification of seizure activity by electrical stimulation. II. Mot seizure Electroencephalogr Clin Neurophysiol. 1972;32:281-94.

20. Xu T, Yu X, Deng J, Ou S, Liu X, Wang T, et al. CXCR7 regulates epileptic seizures by controlling the synaptic activity of hippocampal granule cells. Cell Death Dis. 2019;10:825.

21. Arabadzisz D, Antal K, Parpan F, Emri Z, Fritschy JM. Epileptogenesis and chronic seizures in a mouse model of temporal lobe epilepsy are associated with distinct EEG patterns and selective neurochemical alterations in the contralateral hippocampus. Exp Neurol. 2005;194:76-90.

22. Yang Y, Zhu B, Zheng F, Li Y, Zhang Y, Hu Y, et al. Chronic metformin treatment facilitates seizure termination. Biochem Biophys Res Commun. 2017:484:450-5.

23. Häussler U, Rinas K, Kilias A, Egert U, Haas CA. Mossy fiber sprouting and pyramidal cell dispersion in the hippocampal CA2 region in a mouse model of temporal lobe epilepsy. Hippocampus. 2016;26:577-88.

24. Yuan J, Huang $\mathrm{H}$, Zhou X, Liu X, Ou S, Xu T, et al. MicroRNA-132 interact with p250GAP/ Cdc42 pathway in the hippocampal neuronal culture model of acquired epilepsy and associated with epileptogenesis process. Neural Plast. 2016;2016:5108489.

25. Sah N, Rajput SK, Singh JN, Meena CL, Jain R, Sikdar SK, et al. L-pGlu-(2-propyl)-LHis-L-ProNH $\mathrm{N}_{2}$ attenuates 4-aminopyridine-induced epileptiform activity and sodium current: a possible action of new thyrotropin-releasing hormone analog for its anticonvulsant potential. Neuroscience. 2011;199:74-85.

26. Motamedi GK, Gonzalez-Sulser A, Dzakpasu R, Vicini S. Cellular mechanisms of desynchronizing effects of hypothermia in an in vitro epilepsy model. Neurotherapeutics. 2012;9:199-209.

27. Chijiwa T, Mishima A, Hagiwara M, Sano M, Hayashi K, Inoue T, et al. Inhibition of forskolin-induced neurite outgrowth and protein phosphorylation by a newly synthesized selective inhibitor of cyclic AMP-dependent protein kinase, N-[2-(pbromocinnamylamino) ethyl]-5-isoquinolinesulfonamide (H-89), of PC12D pheochromocytoma cells. J Biol Chem. 1990;265:5267-72. 
28. Huang H, Li R, Yuan J, Zhou X, Liu X, Ou S, et al. Up-regulated ephrinB3/EphB3 expression in intractable temporal lobe epilepsy patients and pilocarpine induced experimental epilepsy rat model. Brain Res. 2016;1639:1-12.

29. Kwan P, Arzimanoglou A, Berg AT, Brodie MJ, Allen Hauser W, Mathern G, et al. Definition of drug resistant epilepsy: consensus proposal by the ad hoc task force of the ILAE commission on therapeutic strategies. Epilepsia. 2010;51:1069-77.

30. Schürmann B, Bermingham DP, Kopeikina KJ, Myczek K, Yoon S, Horan KE, et al. A novel role for the late-onset Alzheimer's disease (LOAD)-associated protein Bin1 in regulating postsynaptic trafficking and glutamatergic signaling. Mol Psychiatry. 2020;25:2000-16.

31. Sheng M, Hoogenraad CC. The postsynaptic architecture of excitatory synapses: a more quantitative view. Annu Rev Biochem. 2007;76:823-47.

32. Scheefhals N, MacGillavry HD. Functional organization of postsynaptic glutamate receptors. Mol Cell Neurosci. 2018;91:82-94.

33. Gao M, Jin Y, Yang K, Zhang D, Lukas RJ, Wu J. Mechanisms involved in systemic nicotine-induced glutamatergic synaptic plasticity on dopamine neurons in the ventral tegmental area. J Neurosci. 2010;30:13814-25.

34. Cabanero D, Baker A, Zhou S, Hargett GL, Irie T, Xia Y, et al. Pain after discontinuation of morphine treatment is associated with synaptic increase of GluA4-containing AMPAR in the dorsal horn of the spinal cord. Neuropsychopharmacology. 2013;38:1472-84.

35. Lu X, Yang Y, Zhou R, Li Y, Yang Y, Wang X. Protrudin modulates seizure activity through $\operatorname{GABA}(\mathrm{A})$ receptor regulation. Cell Death Dis. 2019;10:897.

\section{ACKNOWLEDGEMENTS}

We sincerely thank the patients for their participation. We sincerely thank the Xinqiao Hospital of the Third Military Medical University and the First Affiliated Hospital of Chongqing Medical University for supplying the brain tissues.

\section{AUTHOR CONTRIBUTIONS}

TX, YW, JXY, and YMC designed the study. TX, YW, JXY, and XYY conducted the experiments. TX, YW, JXY, and XYY performed data collection. TX, YW, XL, and CHT performed data analysis. TX and YW drafted the manuscript.

\section{FUNDING}

This study was supported by the National Natural Science Foundation of China (Nos. 81901315,81901330 , and 81771390) and supported by the Kuanren Talents Program of the Second Affiliated Hospital of Chongqing Medical University (No. kryc-yq-2123), China.

\section{COMPETING INTERESTS}

The authors declare no competing interests.

\section{ETHICS STATEMENT}

All animal experiments were approved by the Committee on Animal Research of Chongqing Medical University. The human study complied with the Declaration of Helsinki and was approved by the Committee on Human Research of the Second Affiliated Hospital of Chongqing Medical University.

\section{ADDITIONAL INFORMATION}

Supplementary information The online version contains supplementary material available at https://doi.org/10.1038/s41419-021-04233-2.

Correspondence and requests for materials should be addressed to Tao Xu.

Reprints and permission information is available at http://www.nature.com/ reprints

Publisher's note Springer Nature remains neutral with regard to jurisdictional claims in published maps and institutional affiliations.

\begin{abstract}
(c) Open Access This article is licensed under a Creative Common Attribution 4.0 International License, which permits use, sharing adaptation, distribution and reproduction in any medium or format, as long as you give appropriate credit to the original author(s) and the source, provide a link to the Creative Commons license, and indicate if changes were made. The images or other third party material in this article are included in the article's Creative Commons license, unless indicated otherwise in a credit line to the material. If material is not included in the article's Creative Commons license and your intended use is not permitted by statutory regulation or exceeds the permitted use, you will need to obtain permission directly from the copyright holder. To view a copy of this license, visit http://creativecommons. org/licenses/by/4.0/.
\end{abstract}

(c) The Author(s) 2021 